\title{
Erratum zu: Aktienbasierte erfolgsabhängige Entlohnung \& Unternehmensbewertung
}

\section{A. Schüler}

Online publiziert: 24. Januar 2018

(C) Schmalenbach-Gesellschaft für Betriebswirtschaft e.V. 2018

\section{Erratum zu:}

Schmalenbachs $\mathbf{Z}$ betriebswirtsch Forsch (2017)

https://doi.org/10.1007/s41471-017-0048-7

In der Originalveröffentlichung wurde eine Formel nicht korrekt gesetzt:

Statt:

$$
s_{\mathrm{FCF}}=\frac{\mathrm{FCF}_{U}-\mathrm{FCF}_{D}}{\mathrm{FCF}_{D}}=\frac{\mathrm{FCF}_{U}}{\mathrm{FCF}_{D}}-1
$$

muss es richtig heißen:

$$
s_{\mathrm{FCF}}=\frac{\mathrm{FCF}_{U}-\mathrm{FCF}_{D}}{\mathrm{FCF}_{U}}=1-\frac{\mathrm{FCF}_{D}}{\mathrm{FCF}_{U}}
$$

Wir bitten, diesen Satzfehler zu entschuldigen.

Die Originalveröffentlichung wurde korrigiert.

Die Online-Version des Originalartikels ist unter https://doi.org/10.1007/s41471-017-0048-7 zu finden.

A. Schüler $(\bowtie)$

Fakultät WOW, Universität der Bundeswehr München, Werner-Heisenberg-Weg 39, 85577 Neubiberg,

Deutschland

E-Mail: andreas.schueler@unibw.de 